\title{
Id MM Vaccine Test
}

National Cancer Institute

\section{Source}

National Cancer Institute. Id MM Vaccine Test. NCI Thesaurus. Code C29111.

A test consisting of a myeloma idiotype (Id)-specific protein conjug ated to the immunogen keyhole limpet hemocyanin (KLH). As a test of innate host delayed-type hypersensitivity (DTH) and host DTH in response to therapeutic vaccination, Id MM vaccine test is performed intradermally before and after therapeutic vaccination in multiple myeloma patients treated with Id-specific vaccines. ( $\mathrm{NCl04)}$ 